\title{
Optical Fibre Capacity Optimisation via Continuous Bandwidth Amplification and Geometric Shaping
}

\author{
Lídia Galdino, Member, IEEE, Adrian Edwards, Wenting Yi, Eric Sillekens, Student Member, IEEE, \\ Yuta Wakayama, Thomas Gerard, Student Member, IEEE, Wayne Pelouch, Stuart Barnes, \\ Takehiro Tsuritani, Member, IEEE, Robert I. Killey, Senior Member, IEEE, Domaniç Lavery, Member, IEEE, and \\ Polina Bayvel, Fellow, IEEE
}

\begin{abstract}
The maximum data throughput in a single mode optical fibre is a function of both the signal bandwidth and the wavelength-dependent signal-to-noise ratio (SNR). In this paper, we investigate the use of hybrid discrete Raman \& rare-earth doped fibre amplifiers to enable wide-band signal gain, without spectral gaps between amplification bands. We describe the widest continuous coherent transmission bandwidth experimentally demonstrated to date of $16.83 \mathrm{THz}$, achieved by simultaneously using the S-, C- and L-bands. The variation of fibre parameters over this bandwidth, together with the hybrid amplification method result in a significant SNR wavelengthdependence. To cope with this, the signal was optimised for each SNR, wavelength and transmission band. By using a system-tailored set of geometrically shaped constellations, we demonstrate the transmission of $660 \times 25$ GBd channels over $40 \mathrm{~km}$, resulting in a record single mode fibre net throughput of 178.08 Tbit/s.
\end{abstract}

Index Terms-Broadband transmission system, high order modulation format, geometric shaping.

\section{INTRODUCTION}

$\mathbf{S}$ EVERAL milestones in the achievable data throughput in single mode optical fibres (SMF) [1]-[10] have been reported over the past few years. Fig. 1 shows these record results demonstrated using a range of different amplification techniques. These include distributed Raman amplification, erbium doped fibre amplifiers (EDFA), semiconductor optical amplifiers (SOA), and combinations thereof. Aside from [7], where a capacity of $74 \mathrm{Tbit} / \mathrm{s}$ over $6300 \mathrm{~km}$ was achieved using a hybrid distributed Raman/EDFA (HRE) amplification scheme, all trans-Atlantic $(>6000 \mathrm{~km})$ and trans-Pacific $(>9000 \mathrm{~km})$ record data rates to date have been reached by using $\mathrm{C}+\mathrm{L}$ band EDFAs [10]. Despite HRE schemes having a lower noise figure compared with EDFAs, this amplification technology is not as power efficient as EDFA systems, which makes it less attractive for long-haul submarine systems that are electrical power feed constrained. In these long-distance

This work was supported by UK EPSRC Program Grant TRANSNET EP/R035342/1 and the Royal Academy of Engineering under the Research Fellowships programme and the Royal Society Research Grant.

L. Galdino, W. Yi, E. Sillekens, T. Gerard, D. Lavery, R. I. Killey and P. Bayvel are with the Optical Networks Group, Department of Electronic and Electrical Engineering, UCL, Torrington Place, London WC1E 7JE, UK. e-mail: \{l.galdino; uceewyi; e.sillekens; uceetmh; d.lavery; r.killey; p.bayvel\}@ucl.ac.uk.

A. Edwards, W. Pelouch, and S. Barnes, are with Xtera, Bates House, Church Road, Harold Wood, Essex, UK. e-mail: \{adrian.edwards; wayne.pelouch; stuart.barnes\}@xtera.com.

Y. Wakayama and T. Tsuritani are with KDDI Research, 2-1-15 Ohara, Fujimino 356-8502, Japan, email: \{yu-wakayama; tsuri $\}$ kddi-research.jp. Manuscript received xxx; revised xx, 2020

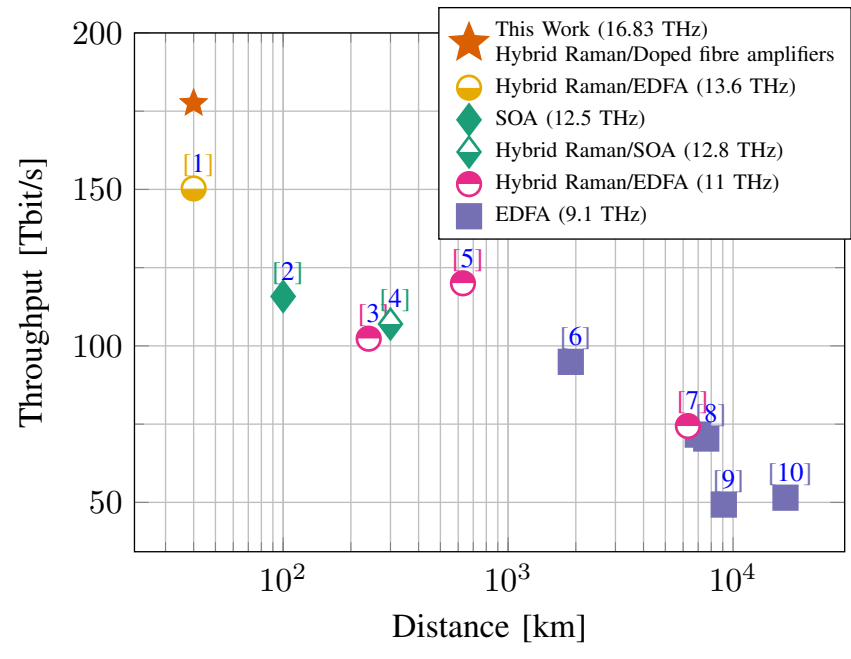

Fig. 1. Record data throughput versus distance for single mode fibre. The transmission bandwidth indicated in each case is for the usable spectrum, not including spectral gaps between amplifier gain bandwidths.

transmission systems, advanced coded modulation schemes and nonlinearity compensation algorithms were used to increase both the throughput and reach. In contrast, record capacities over short transmission distances have mainly used amplification technologies that extend beyond C+L-band EDFAs. In [2], an SOA with a $100 \mathrm{~nm}$ gain bandwidth demonstrated the potential for an SMF capacity of $115.9 \mathrm{Tbit} / \mathrm{s}$ over a $100 \mathrm{~km}$ transmission distance. The relatively high noise figure of SOAs versus, for example, EDFAs means they are generally considered unsuitable for repeatered transmission systems. Nevertheless, by combining an SOA with distributed backward Raman amplification, 107 Tbit/s transmission over $300 \mathrm{~km}(3 \times 100 \mathrm{~km})$ was reported in [4]. A yet greater data rate of $120 \mathrm{Tbit} / \mathrm{s}$ over $630 \mathrm{~km}(9 \times 70 \mathrm{~km})$ was shown by using hybrid distributed Raman-EDFA amplifiers with a continuous $91 \mathrm{~nm}$ gain bandwidth [5].

Extending the transmission bandwidth to S-band wavelengths resulted in the highest single mode fibre capacity experimentally shown to date of $150.3 \mathrm{Tbit} / \mathrm{s}$, transmitted over $40 \mathrm{~km}$ [1] This experiment [1] used a distributed backward Raman amplification scheme for the S-band and EDFAs for C- and L-bands; with the transmitted signal occupying a total bandwidth of approximately $109 \mathrm{~nm}(13.625 \mathrm{THz})$. However, the gain bandwidth was not continuous, with approximately 17 and $5 \mathrm{~nm}$ spectral gaps between S/C- and C/L-bands, respectively. This reduces the spectral efficiency and diminishes the benefit of transmitting signals in multiple fibre transmission 


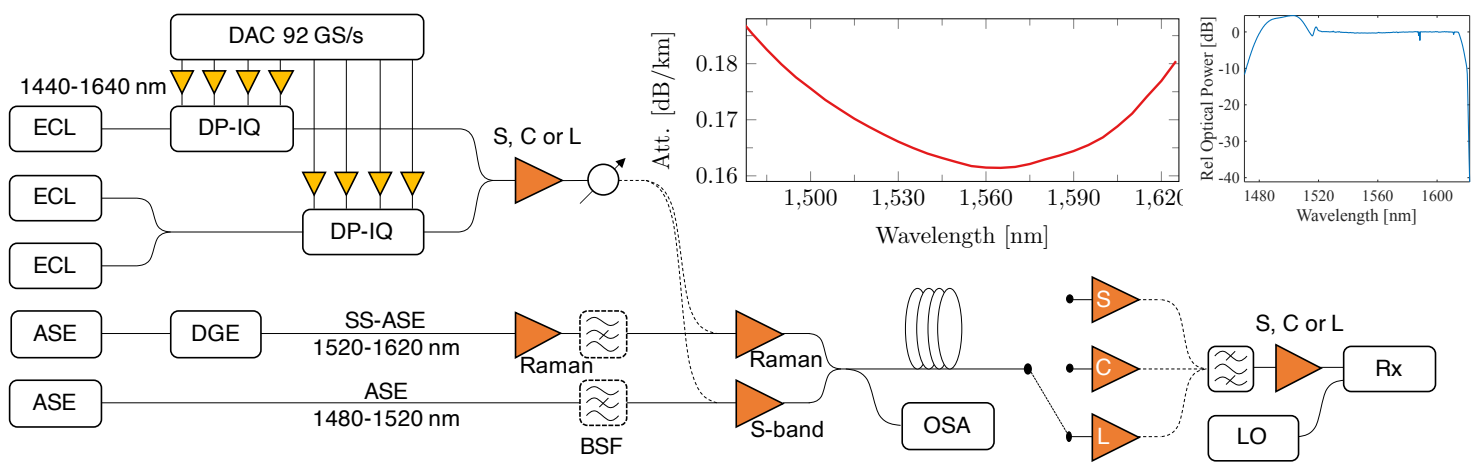

Fig. 2. Schematic of the transmission experiment. Inset: optical fibre attenuation profile and signal spectrum before launching into the optical fibre (the modulated channels are centered at $1588 \mathrm{~nm}$ ). Key: Dynamic Gain Equaliser (DGE), Spectrally Shaped Amplified Spontaneous Emission Noise (SS-ASE), Bandstop Filter (BSF).

windows.

In this paper, we describe the use of a combination of discrete Raman amplifiers with a continuous gain bandwidth of 1520.00-1619.67 nm, together with a thulium doped fibre amplifier (TDFA), 1484.86-1520.00 nm gain bandwidth, to demonstrate the widest continuous coherent transmission bandwidth of $16.83 \mathrm{THz}$ reported to date in a single fibre core. The use of the combination of amplifier technologies led to a wavelength-dependent SNR variation. To increase the overall data throughput, we therefore designed and implemented new, geometrically-shaped (GS) constellations, tailored for the SNR of each wavelength, maximising the achievable information rate (AIR) of each individual channel. Pilot-based digital signal processing was used, and the AIR was estimated by deducting the pilot overhead $(\mathrm{OH})$ from the per channel generalised mutual information (GMI), assuming an additive white Gaussian noise (AWGN) auxiliary channel [11, Eq. (53)]. The overall net throughput of $178.08 \mathrm{Tbit} / \mathrm{s}$ reported represents a record data rate for single core, single mode optical fibre transmission.

\section{EXPERIMENTAL TRANSMISSION SET UP}

The experimental configuration is shown in Fig. 2. Three tuneable external cavity lasers (ECL), spaced by $25.5 \mathrm{GHz}$, were connected to two independent dual-polarisation IQ optical modulators, each driven by $92 \mathrm{GS} / \mathrm{s}$ digital-to-analogue converters (DAC) to generate three odd/even channels. A digital root-raised cosine (RRC) filter ( 0.01 roll-off) was used to spectrally shape the signals. The channels were generated at carrier frequencies which were tuned across the range $1484.86-1619.67 \mathrm{~nm}$, allowing the measurement of $660 \times 25 \mathrm{GBd}$ dual-polarisation signals. The modulated channels were amplified using TDFA, C/L band EDFA and/or discrete Raman amplifiers, as appropriate for the wavelength of the channel under test. The three channels were combined with wideband amplified spontaneous emission (ASE) noise, which emulated co-propagating channels, followed by a band stop filter (BSF) for creating a spectral gap at the location where the modulated channels were inserted. The validity of using ASE noise to emulate aggressor channels was verified in [12], showing that this technique provides a conservative measure of system performance.The modulated channel power was adjusted to be the same as the surrounding ASE. The ASE noise, generated by a pair of discrete Raman amplifiers to achieve a total output power of $21.5 \mathrm{dBm}$ over the entire bandwidth (from 1520.00-1619.67 nm), was coupled with ASE noise generated by TDFAs with a noise bandwidth of $40 \mathrm{~nm}$ (from 1470-1520.00 nm) and a total output power of $19 \mathrm{dBm}$. Note that due to a sharp decrease on the responsivity of the balanced photo-detectors at lower wavelengths the lowest wavelength received was $1484.86 \mathrm{~nm}$.

A dynamic gain equaliser (DGE) with a continuous $100 \mathrm{~nm}$ bandwidth was used to spectrally shape the ASE noise generated by the discrete Raman amplifiers with wavelength bandwidth between 1520 and $1620 \mathrm{~nm}$. The S-band ASE noise was not shaped as a gain equaliser for this wavelength range was not available for the experiment; however, an S/C band WDM splitter was placed after the S-band ASE noise source, producing a sharp roll-off of the ASE at approximately $1520.00 \mathrm{~nm}$. The combined ASE noise and modulated channels occupied a total usable bandwidth of $16.83 \mathrm{THz}$ $(134.81 \mathrm{~nm})$ with a total output power of $20.4 \mathrm{dBm}$.

A $40 \mathrm{~km}$ Corning ${ }^{\circledR}$ SMF - $28^{\circledR}$ ULL fibre span with $0.16 \mathrm{~dB} / \mathrm{km}$ attenuation at C-band, and an additional $0.4 \mathrm{~dB}$ loss on the fibre output due to splicing and connectorisation, was used as the transmission span. Its fibre attenuation vs wavelength is shown inset Fig. 2. A TDFA, EDFA or discrete Raman amplifier was used to compensate the fibre loss, depending on the spectral range. A TDFA with $7 \mathrm{~dB}$ NF was used for wavelength channels between 1484.86 - 1519.8 $\mathrm{nm}$. For channels spaced in the 1520 - $1529 \mathrm{~nm}$ and 1608 $1619.67 \mathrm{~nm}$ ranges a discrete Raman amplifier with average noise figure of $9 \mathrm{~dB}$ was used. $\mathrm{C}$ and L-band EDFAs with 5.5 and $6 \mathrm{~dB} \mathrm{NF}$, respectively, were used to amplify the remaining bandwidth of 1529.2 - $1607.8 \mathrm{~nm}$. At the receiver side, the signal was filtered by using an optical band pass filter (BPF) and amplified before being sent to the coherent receiver. Due to the limited number of TDFAs available, two cascaded SOAs were used to boost the S-band signal power to the receiver. The optical coherent receiver was composed of a polarisation and phase diverse optical hybrid and four balanced photodiodes with a bandwidth of $65 \mathrm{GHz}$. A digital-storage oscilloscope (DSO) with a bandwidth of $110 \mathrm{GHz}$ digitised the received $25 \mathrm{GBd}$ signal at a sampling rate of $256 \mathrm{GS} / \mathrm{s}$. 


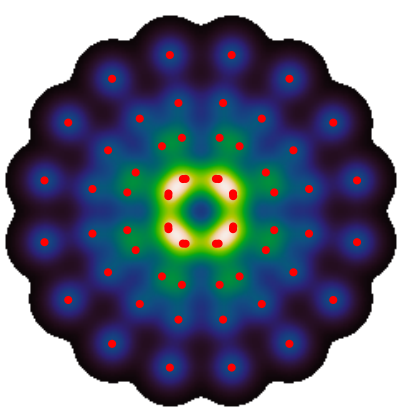

(a)

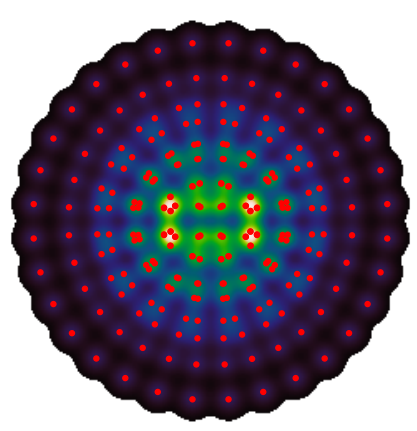

(b)

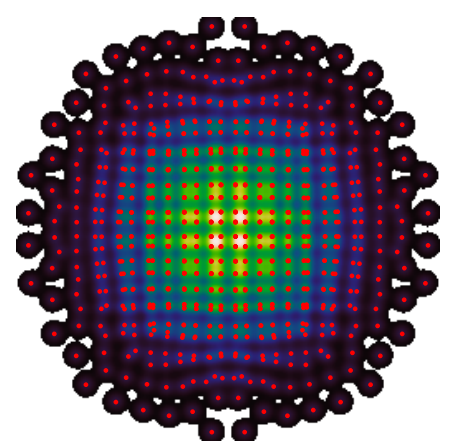

(c)

Fig. 3. Constellation diagrams of: GS-64QAM for an SNR of $12 \mathrm{~dB}$ (a), GS-256QAM for an SNR of $16 \mathrm{~dB}$ (b), and GS-1024QAM for an SNR of 20 dB (c). The pink markers illustrate the generated constellation diagram. Constellation coordinates and bit-to-symbol mappings are available for download at [15].

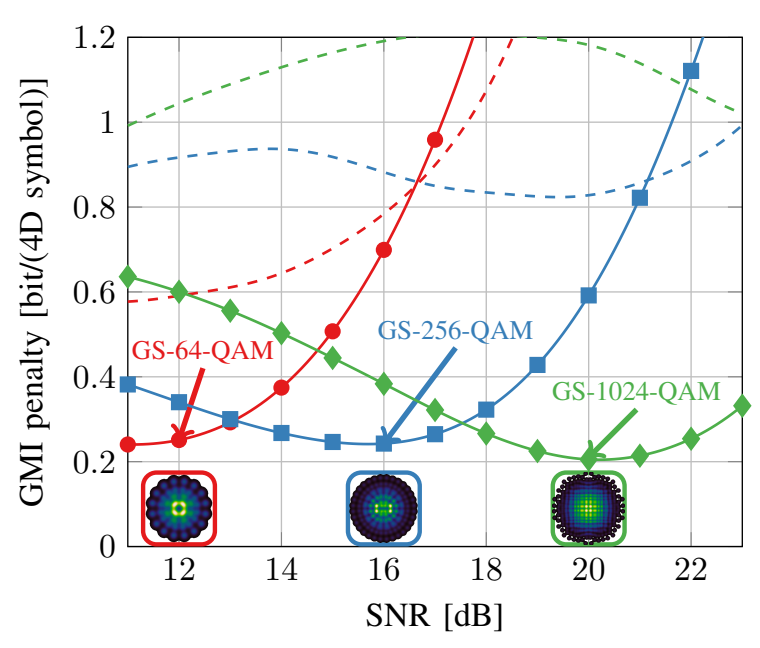

Fig. 4. Gap to AWGN capacity for each modulation format. The target SNRs for which each of the constellations were optimised are indicated by the arrows. Dashed lines show the gap to capacity for square QAM constellations. Constellation coordinates and bit-to-symbol mappings are available at [15].

Pilot-based digital signal processing was implemented as described in [13], with QPSK pilot symbols embedded within the data payload instead of using a separate optical pilot tone. This facilitates equalisation and carrier recovery for high-order and/or non-uniform constellations in a standard intradyne configuration, and was key to recovering the information for any designed GS constellation without incurring any SNR penalty. Mersenne twister random integer sequence with $2^{16}$ length was used for the payload and pilot symbols. The required pilot overhead $(\mathrm{OH})$ was optimised to maximise the overall AIR [13]. Pilot symbols for carrier phase estimation were inserted at a rate of $1 / 32$. The pilot sequence length and the frame length were fixed at 1024 symbols and 65536 symbols, respectively, thus requiring a pilot $\mathrm{OH}$ of $4.64 \%$.

\section{Geometric Constellation Shaping}

The GS formats were designed by using a gradient descent algorithm with a cost function seeking to maximise GMI at the given received SNR (assuming a linear AWGN channel), as per [14]. For the transmission system under test, the received signal SNR varied between $12 \mathrm{~dB}$ and $20 \mathrm{~dB}$ over the bandwidth

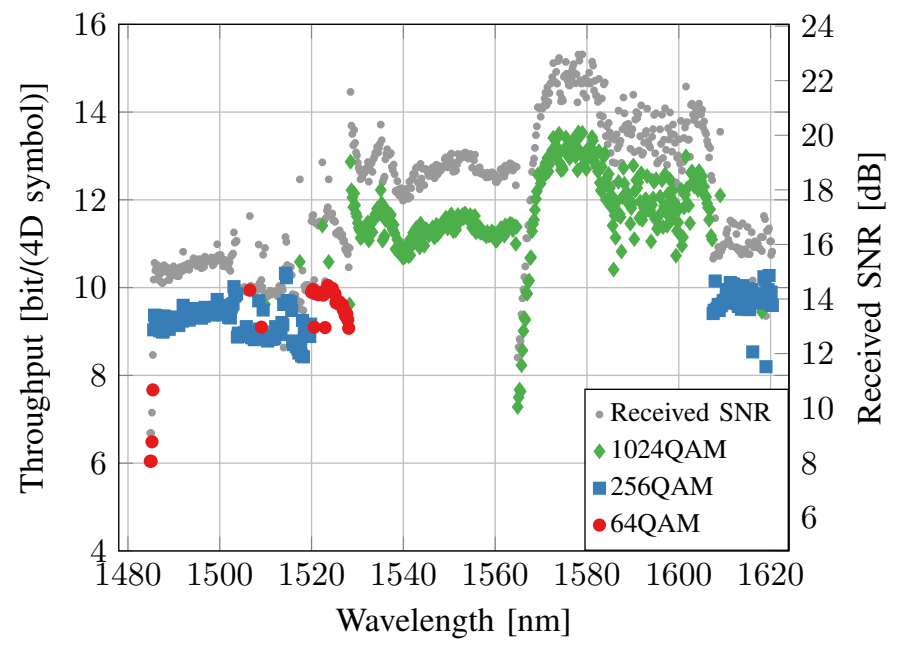

Fig. 5. Per-channel throughput over 2 polarisations after $40 \mathrm{~km}$.

due to the combination of the variation of fibre parameters over this wavelength range and the amplifier noise figures and gain profiles. Thus, to maximise the AIR of each channel, GS modulation formats of 64, 256 and 1024 constellation points were designed for a received SNR between 12 and $23 \mathrm{~dB}$. Fig. 3 shows the designed GS-64QAM, GS-256QAM and GS1024QAM constellations for a received SNR of 12, 16 and $20 \mathrm{~dB}$, respectively. (Here, GS-MQAM is taken to mean $M$-ary constellations, of equal probability, shaped in two dimensions.) The pink markers display the generated constellation diagram. The coordinates of each constellation are available at [15].

Fig. 4 illustrates the AIR gap for each optimised GS constellation to AWGN capacity for received SNR of 12, 16 and $20 \mathrm{~dB}$. The dashed lines show the AIR gap to AWGN capacity for square 64QAM (red), 256QAM (blue) and 1024QAM (green). The red circles show the optimised GS-64QAM constellation for $12 \mathrm{~dB}$ SNR, while the blue square and green triangle markers illustrate the optimised GS256QAM and GS-1024QAM constellations for 16 and $20 \mathrm{~dB}$ SNR, respectively.

It can be seen that, through the combination of different GS-MQAM optimisations, the GMI gap to AWGN capacity can be maintained below $0.3 \mathrm{bit} /(4 \mathrm{D}$ symbol) for the target 
SNRs. For SNRs below $13 \mathrm{~dB}$, the optimised GS-64QAM has a lower GMI penalty in comparison to the higher-order carnality GS constellations. Similarly, for SNRs between 13 and $17.8 \mathrm{~dB}$, GS-256QAM is needed to maintain the GMI gap below $0.3 \mathrm{bit} /(4 \mathrm{D}$ symbol). For an SNR greater than $17.8 \mathrm{~dB}$, GS-1024QAM should be used. Finally, we also note that the GS constellations exhibit an improvement in GMI compared to the square QAM formats. For instance, the GMI improvement of GS-64QAM compared to 64QAM at $12 \mathrm{~dB}$ SNR is 0.34 bit/4D-symbol, while the GMI improvement of GS-256QAM at $16 \mathrm{~dB}$ and GS-1024QAM at $20 \mathrm{~dB}$ are $0.64 \mathrm{bit} / 4 \mathrm{D}$-symbol and $0.98 \mathrm{bit} / 4 \mathrm{D}$-symbol, respectively.

Note that the square 256QAM constellation has a mean GMI gap of $0.9 \mathrm{bit} / 4 \mathrm{D}$ symbol across the range of SNRs considered here. This gap is $0.6 \mathrm{bit} / 4 \mathrm{D}$ symbol higher than the designed GS-MQAM constellation. Therefore, with the use of geometric constellation shaping in this experiment, the data throughput was estimated to be $9.5 \mathrm{Tbit} / \mathrm{s}$ higher than would be possible with square 256QAM.

We have also designed GS constellations for all SNR values (in $1 \mathrm{~dB}$ steps). We observed that the GMI gap would have been maintained below $0.2 \mathrm{bit} / 4 \mathrm{D}$ symbol for all wavelength channels, compared with a GMI gap below 0.3 bit/4D symbol for 3 optimised constellations. If designed GS constellations for each SNR value were used, only a marginal gain of $1.5 \mathrm{Tbit} / \mathrm{s}$ would have been observed.

\section{PERformance Analysis}

The information rate and SNR are shown in Fig. 5 for all the $660 \times 25 \mathrm{GBd}$ channels received after the $40 \mathrm{~km}$ transmission. The per channel throughput, in bit/symbol over both polarisations, was calculated by multiplying the mean AIR, over both polarisations, by the channel symbol rate. As previously noted, the GS modulation formats were tailored to maximise AIR for each value of the received SNR. The mean received SNR was $20.36 \mathrm{~dB}$, with the measured values in the range 9.6-23.0 dB over the entire transmission bandwidth. $99 \%$ of channels had SNRs greater than $13 \mathrm{~dB}$. As can be seen in Fig. 5, the lower AIRs (and, thus, SNRs) were in the S-band, due to the lack of a DGE and the use of cascaded SOAs to pre-amplify the received signal. For these channels, optimum performance was generally achieved using 64-ary or GS 256-ary GS, with an average AIR of 10.79 bit/symbol.

In the C- and L-bands, 1024-ary GS constellations were mainly used. Note that, although there was some SNR degradation between the C- and L-bands, 1024-ary GS was used for continuity of measurement. It can also be noted that, for channel wavelengths between 1520 and $1529 \mathrm{~nm}$, GS-64QAM was used, despite the fact GS-256QAM provides higher AIR. At these wavelengths, the discrete Raman amplifier used introduced nonlinear distortion to the signal, degrading the received SNR by approximately $7 \mathrm{~dB}$ compared to the data shown in Fig. 5. After offline DSP processing, which included a nonlinear phase-shift compensation, the nonlinear distortion generated by the DRA was mitigated, improving the received SNR. Therefore, if GS-256QAM had been used, higher throughput could potentially have been achieved for these wavelengths.
Judicious selection of modulation format is key in this experiment. As shown in Fig. 4, using a single GS modulation format would have substantially decreased the throughput, due to an increased gap to capacity at either lower or higher SNRs than the target SNR for GS constellations. Combining the measured per-channel GMI, and deducting the pilot overhead, the achievable information rate for this transmission system is an aggregate throughput of $178.08 \mathrm{Tbit} / \mathrm{s}$.

\section{CONCLUSIONS}

In this work, a record transmission throughput for a single core, single mode optical fibre was demonstrated, using a continuous, ultra-wideband $(16.83 \mathrm{THz})$ transmission window. The latter was enabled using hybrid discrete Raman/doped fibre amplification, which, together with the pilot-based DSP, allowed the use of SNR-tailored geometric constellation shaping. The geometric shaping accounted for approximately $9.5 \mathrm{Tbit} / \mathrm{s}$ increase with the overall throughput of $178.08 \mathrm{Tbit} / \mathrm{s}$, over $18 \%$ higher than the previous record of $150.3 \mathrm{Tbit} / \mathrm{s}$ [1].

The authors thank Keysight for the loan of the Infiniium UXR oscilloscope used in this work, Oclaro for the high-bandwidth modulator, and Corning for the loan of the Corning ${ }^{\circledR}$ SMF - $28^{\circledR}$ ULL fiber.

\section{REFERENCES}

[1] F. Hamaoka et al., "150.3-Tb/s Ultra-Wideband (S,C and L bands) Single-Mode Fibre Transmission over 40-km Using $\measuredangle 519 \mathrm{~GB} / \mathrm{s} / \lambda$ PDM128QAM Signals," ECOC, Mo4G.1, (2018).

[2] J. Renaudier et al., "First 100-nm Continuous-Band WDM Transmission System with $115 \mathrm{~Tb} / \mathrm{s}$ Transport over $100 \mathrm{~km}$ Using Novel UltraWideband Semiconductor Optical Amplifiers," ECOC, PDP, (2017).

[3] A. Sano et al., "102.3-Tb/s C-band extended L-band all Raman transmission over $240 \mathrm{~km}$ using PDM-64QAM single carrier FDM with digital pilot tone," OFC, PDPSC.3 (2012).

[4] J. Renaudier et al., "107 Tb/s Transmission of 103-nm Bandwidth over $3 \times 100 \mathrm{~km}$ SSMF using Ultra-Wideband Hybrid Raman/SOA Repeaters", OFC, Tu3F.2, (2019).

[5] L. Galdino, et al., "Study on the Impact of Nonlinearity and Noise on the Performance of High-Capacity Broadband Hybrid Raman-EDFA Amplified System", J. Lightw. Technol., 37 (21), (2019).

[6] J.-X. Cai et al.,"94.9 Tb/s Single Mode Capacity Demonstration over $1,900 \mathrm{~km}$ with C+L EDFAs and Coded Modulation", ECOC, (2018).

[7] M. Ionescu, et al., $74.38 \mathrm{~Tb} / \mathrm{s}$ Transmission Over $6300 \mathrm{~km}$ Single Mode Fibre Enabled by $\mathrm{C}+\mathrm{L}$ Amplification and Geometrically Shaped PDM64QAM J. Lightw. Technol., vol.38 n.2, (2020).

[8] J.-X. Cai et al., "70.46 Tb/s over 7,600 km in in C+ L Band Using Coded Modulation with Hybrid Constellation Shaping and Nonlinearity Compensation," OFC, PDP Th5B.2, (2017).

[9] T-X. Cai et al., "49.3 Tb/s Transmission Over $9100 \mathrm{~km}$ Using C+L. EDFA and $54 \mathrm{~Tb} / \mathrm{s}$ Transmission Over $9150 \mathrm{~km}$ Using Hybrid-Raman EDFA," J. Lightw. Technol., vol.33, n.13, (2015).

[10] J.-X. Cai et al., "51.5 Tbps Capacity over 17,107 km in C+L Bandwidth Using Single-Mode Fibers and Nonlinearity Compensation," J. Lightw. Technol., vol.36, no.11, (2018)

[11] A. Alvarado et al., "Achievable Information Rates for Fiber Optics: Applications and Computations," J. Lightw. Technol., vol.36, no.2, (2018)

[12] J.-X. Cai et al., "On the Effects of Transmitter Induced Channel Correlation in Broadband WDM Transmission," OFC, Th1C.1 (2018).

[13] Y. Wakayama et al., "Increasing Achievable Information Rates with Pilot-Based DSP in Standard Intradyne Detection” ECOC, (2019).

[14] E. Sillekens et al., "Experimental Demonstration of GeometricallyShaped Constellations Tailored to the Nonlinear Fibre Channel," in ECOC, Tu3G.3 (2018).

[15] E. Sillekens, D. Lavery, "Geometrically Optimised Constellation for $12 \mathrm{~dB}, 16 \mathrm{~dB}$ and $20 \mathrm{~dB}$ SNR AWGN channel." Online: doi.org/10.5522/04/11894325 